\title{
Theoretical Analysis of a two-stage Sagnac loop filter Using Jones Matrices
}

\author{
N. A. B. Ahmad ${ }^{1}$, S. H. Dahlan' ${ }^{2}$ N. A. Cholan ${ }^{3}$ \\ ${ }^{1,2,3}$ Research Center for Applied Electromagnetics, Universiti Tun Hussein Onn Malaysia (UTHM), 86400 Parit Raja, \\ Batu Pahat, Johor, Malaysia \\ ${ }^{1,2,3}$ Faculty of Electrical and Electronic Engineering, Universiti Tun Hussein Onn Malaysia (UTHM), 86400 Parit Raja, \\ Batu Pahat, Johor, Malaysia
}

\begin{tabular}{l} 
Article Info \\
\hline Article history: \\
Received Aug 22, 2017 \\
Revised Nov 14, 2017 \\
Accepted Nov 30, 2017 \\
\hline
\end{tabular}

\section{Keyword:}

Multiwavelength fiber laser Optical fiber

Polarization controller (PC)

Polarization maintaining fiber

Sagnac loop filter (SLF)

\begin{abstract}
In this work, a theoretical analysis of a Sagnac loop filter (SLF) with twostage polarization maintaining fibers (PMFs) and polarization controllers (PCs) is presented. The transmission function of this two-stage SLF is calculated in detail by using Jones matrix. The calculation is performed in order to investigate the filtering characteristics. The theoretical results show that the wavelength interval is depending on the dynamic settings of the length of the PMFs and the polarization angle of the PCs. By changing the polarization angle of the PCs, a multiple of single, dual or triple wavelength in each channel can be achieved. Based on this study, a flat multiwavelength spectrum can be obtained by adjusting the PMFs and the PCs in the twostage SLF. This finding significantly contributes to the generation of multiwavelength fiber laser (MWFL) that can be used for many optical applications.
\end{abstract}

Copyright $(9) 2017$ Institute of Advanced Engineering and Science. All rights reserved.

\section{Corresponding Author:}

\section{N. A. B. Ahmad,}

Faculty of Electrical and Electronic Engineering,

Universiti Tun Hussein Onn Malaysia (UTHM),

86400 Parit Raja, Batu Pahat, Johor, Malaysia.

Email: nurulati89@gmail.com

\section{INTRODUCTION}

Many articles on multiwavelength fiber laser (MWFL) have been published leading to the contribution of various applications such as optical instrument testing, optical fiber sensing, spectroscopy, microwave photonics, optical communication networks, and dense wavelength division multiplexing (WDM) system [1-3]. The multiwavelength characteristics in terms of number of lines, channel spacing, wavelength tenability, flatness, and full band coverage were extensively discovered [4]. In recent years, most of MWFL researches are based on the use of erbium-doped fiber amplifier (EDFA) due to its many advantages such as low polarization sensitivity, high saturation power, wide bandwidth and broadband gain in the communication window [5-7]. However, owing to the EDFA homogeneous line broadening, it is difficult to achieve stable MWFL at room temperature. Another popular gain mechanism for MWFL is Raman amplification which is particularly attractive due to low noise and its ability to be generated at any wavelength (dependent on pump wavelength) without the need for a specialized gain medium but high pump power is required to operate this laser [8], \{9].

A common method of generating MWFL is through the use of nonlinear effects such as stimulated Brillouin scattering (SBS) [10], four-wave mixing (FWM), and nonlinear polarization rotation (NPR) [11]. NPR has emerged as an interesting choice due to its advantages of performing changeable operating regimes of multiwavelength lasing as well as passively mode locked at deploying a comb filter within the laser 
structure. The comb filter which was based on the Sagnac loop filter (SLF) had been used extensively in the design of MWFL [12-14].

SLF is popular to be used for various applications due to its simple structure and output variability which can simply be done by varying the PC and PMF parameter [15-17]. Tuning characteristic is a desired feature of any comb filter when it is used in a multiwavelength light source. The SLF of one-stage polarization-maintaining fiber (PMF) and polarization controller (PC) is an example of a simple filter device as used in [15]. In that SLF, the PC consists only of two waveplates and the wavelength tunability is realized by tuning the two waveplates. In [16], two PCs in the SLF was reported where each PC is located at both ends of the PMF.

In this paper, a two-stage Sagnac loop filter (SLF) consist of two polarization maintaining fibers (PMFs) and two polarization controllers (PCs) were investigated and demonstrated theoretically. The operating principle is studied thoroughly and simulated by applying the Jones matrix. To characterize this two-stage SLF, we vary the length of the PMFs and the polarization angle of the PCs. The wavelength interval depends on both PMFs and PCs was confirmed by the transmission spectrum results. The multiple single, dual or triple wavelength lasing are obtained by adjusting the PCs.

\section{THEORETICAL AND OPERATING PRINCIPLE}

The structure of two polarization maintaining fibers (PMFs) and two polarization controllers (PCs) of Sagnac loop filter (SLF) is schematically shown in Figure 1. PMF1 and PMF2 are two PMFs with different lengths and birefringence. PC1 and PC2 are two PCs made from a half-wave plate (HWP) coil and two quarter-wave plates (QWP) coils at both ends. They are alternately connected for the two-stage SLF. Every stage consists of one PMF and one PC. A polarization-insensitive $3 \mathrm{~dB}$ coupler is used to connect the ring cavity and the two-stage SLF. The lights travel into the $3 \mathrm{~dB}$ coupler through port 1 , and then splits into two. Fifty percent of the output lights travel clockwise $(\mathrm{CW})$ around the loop through port 3 . Meanwhile, the other fifty percent of the output lights travel counterclockwise (CCW) with $\pi / 2$ phase difference through port 4. The two lights contrarily pass through the PCs and PMFs before reentering the $3 \mathrm{~dB}$ coupler and transmitted out through port 2.

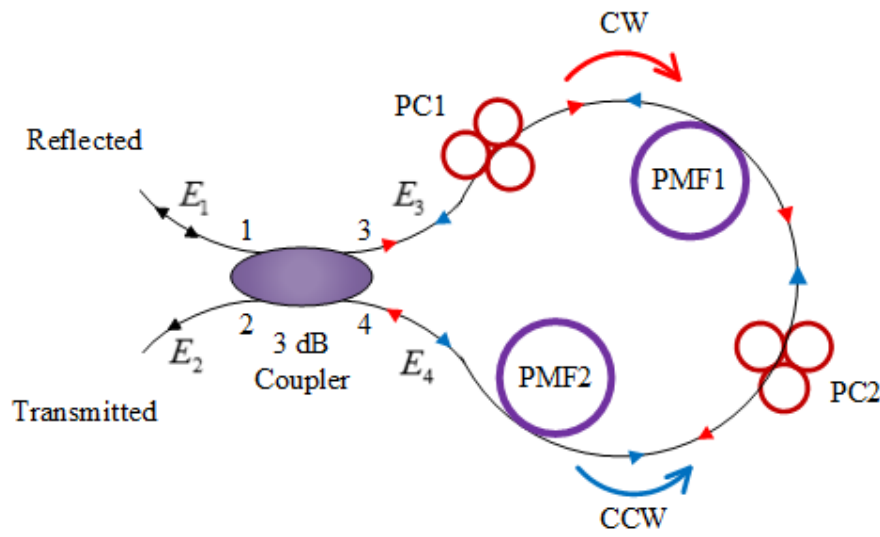

Figure 1. The structure of two-stage Sagnac loop filter (SLF) for the analysis of Jones matrices formulism

The polarization state of the $\mathrm{CW}$ and CCW travelling lights in this work was analyzed using Jones matrix [17] which can describe efficiently the polarization state of a plane wave.

In general, electric field can be expressed in its complex amplitude

$$
E=\left[\begin{array}{c}
E_{X} \\
E_{Y}
\end{array}\right]=\left[\begin{array}{l}
E \cos \theta \\
E \sin \theta
\end{array}\right]
$$

where $\theta$ is the angle in between the polarization direction of the input light with the $\mathrm{x}$-axis. Note that there are CW light propagations (from port 3 to port 4), and CCW light propagations (from port 4 to port 3 ). Thus, there are four electric fields going in and out of the Sagnac loop filter. The initial lights are coupled into the 
fiber loop by the $3 \mathrm{~dB}$ coupler. The fields at port 1 and 2 can be related to the fields at port 3 and 4 using the Jones matrix (represented by $M_{C}$ ), as described in [18]

$$
\left[\begin{array}{l}
E_{3} \\
E_{4}
\end{array}\right]=M_{C}\left[\begin{array}{l}
E_{1} \\
E_{2}
\end{array}\right]
$$

$E_{3}$ and $E_{4}$ are the output electric fields of port 3 and port 4, and $E_{1}$ and $E_{2}$ are the initial electric fields of port 1 and port 2 as shown in Figure 1. The initial light is input only to the Port 1 , hence $E_{2}=0$. The CW light out of port 3 goes through the PC first. The Jones matrix for HWP and QWPs of the PC is as follows [19]

$$
T\left(\theta_{j}\right)=\left[\begin{array}{cc}
\cos \frac{\varphi_{j}}{2}+i \sin \frac{\varphi_{j}}{2} \cos 2 \theta_{j} & i \sin \frac{\varphi_{j}}{2} \sin 2 \theta_{j} \\
i \sin \frac{\varphi_{j}}{2} \sin 2 \theta_{j} & \cos \frac{\varphi_{j}}{2}-i \sin \frac{\varphi_{j}}{2} \cos 2 \theta_{j}
\end{array}\right]
$$

where $\theta_{\mathrm{j}}$ is the angular orientations of the three waveplates, with $j$ is the waveplates index, 1,2 , and $3 . \varphi_{\mathrm{j}}$ denoted as $\varphi_{1}=2 \pi / m, \varphi_{2}=2 \pi / l$, and $\varphi_{3}=2 \pi / n$ where the $l, m$ and $n$ is the number of loop in the PC plates which is equal to 4,2 , and 4 , respectively. When the lights travel from port 3 to port 4 , they go through the $\mathrm{PC}$ in sequence of QWP-HWP-QWP.

The Jones matrix for PC1 and PC2 are representation of the product of three Jones matrices

$$
\begin{aligned}
& M_{P C 1}\left(\theta_{11}, \theta_{12}, \theta_{13}\right)=\frac{i}{2}\left(\begin{array}{cc}
A+i B & C+i D \\
C-i D & -A+i B
\end{array}\right) \\
& M_{P C 2}\left(\theta_{21}, \theta_{22}, \theta_{23}\right)=\frac{i}{2}\left(\begin{array}{cc}
E+i F & G+i H \\
G-i H & -E+i F
\end{array}\right)
\end{aligned}
$$

where,

$$
\begin{aligned}
& A=\cos \left(2 \theta_{12}\right)-\cos \left(2 \theta_{13}-2 \theta_{12}+2 \theta_{11}\right) \\
& B=\cos \left(2 \theta_{13}-2 \theta_{12}\right)+\cos \left(2 \theta_{12}-2 \theta_{11}\right) \\
& C=\sin \left(2 \theta_{12}\right)-\sin \left(2 \theta_{13}-2 \theta_{12}+2 \theta_{11}\right) \\
& D=\sin \left(2 \theta_{13}-2 \theta_{12}\right)+\sin \left(2 \theta_{12}-2 \theta_{11}\right) \\
& E=\cos \left(2 \theta_{22}\right)-\cos \left(2 \theta_{23}-2 \theta_{22}+2 \theta_{21}\right) \\
& F=\cos \left(2 \theta_{23}-2 \theta_{22}\right)+\cos \left(2 \theta_{22}-2 \theta_{21}\right) \\
& G=\sin \left(2 \theta_{22}\right)-\sin \left(2 \theta_{23}-2 \theta_{22}+2 \theta_{21}\right) \\
& H=\sin \left(2 \theta_{23}-2 \theta_{22}\right)+\sin \left(2 \theta_{22}-2 \theta_{21}\right)
\end{aligned}
$$

From (4)-(6), the angles of $\theta_{11}, \theta_{12}$, and $\theta_{13}$ are for the polarization state of PC1, while the angles of $\theta_{21}, \theta_{22}$, $\theta_{23}$ are for the polarization state of $\mathrm{PC} 2$.

The PMF is a high birefringence fiber which causes the phase shift $(\Delta \varphi)$. The Jones matrix of a PMF that depending on $\Delta \varphi$ is represented as

$$
M_{P M F}(\varphi)=\left(\begin{array}{cc}
1 & 0 \\
0 & e^{i \Delta \varphi}
\end{array}\right)
$$

where $\Delta \varphi=2 \pi L \Delta n / \lambda$ is the phase shift of the PMF. $\Delta n$ is the refractive index difference between the fast-axis and the slow-axis of the PMF (birefringence), $L$ is the length of the PMF and $\lambda$ is the wavelength of the propagation light. Consequently, $\Delta \varphi_{1}, \Delta n_{1}$, and $L_{1}$ are parameters referred for PMF1 and $\Delta \varphi_{2}, \Delta n_{2}$, and $L_{2}$ are parameters referred for PMF2.

The Jones Matrices of CW (from port 3 to port 4) and CCW (from port 4 to port 3) of the Sagnac loop filter as the products of $M_{P M F 1}, M_{P M F 2}, M_{P C l}$, and $M_{P C 2}$, are as shown in [20] 


$$
\begin{aligned}
& M_{C W}=M_{P M F 2} \cdot M_{P C 2} \cdot M_{P M F 1} \cdot M_{P C 1} \\
& M_{C C W}=M_{P C 1} \cdot M_{P M F 1} \cdot M_{P C 2} \cdot M_{P M F 2}
\end{aligned}
$$

In this analysis, the electric field $\mathrm{E}_{3}$ at port 3 will go through PC1-PMF1-PC2-PMF2 and denoted as $\mathrm{E}_{4}^{\prime}$ when it reaches port 4. Same notation scheme is used for electric fields travelling from port 4 to port 3 where the field is denoted as $E_{3}^{\prime}$. After looping the fiber, the electric field at port 3 and port 4 can be written as $E_{3}^{\prime}=M_{C C W} \cdot E_{4}^{\prime}$ and $E_{4}^{\prime}=M_{C C W} \cdot E_{3}^{\prime}$. Then $E_{3}^{\prime}$ and $E_{4}^{\prime}$ are coupled into the coupler again and the resulting output lights can be related with its input as

$$
\left[\begin{array}{l}
E_{1}^{\prime} \\
E_{2}^{\prime}
\end{array}\right]=M_{C C W}\left[\begin{array}{l}
E_{3}^{\prime} \\
E_{4}^{\prime}
\end{array}\right]
$$

where $E_{1}^{\prime}$ is the reflected electric field at port 1 and $E_{2}^{\prime}$ is the transmitted electric field at port 2. $M_{C C W}$ is the matrix when lights go through the $3 \mathrm{~dB}$ coupler inversely. Finally, the transmission function for $\mathrm{CW}$ and $\mathrm{CCW}$ propagations can be derived based on (8) as follow

$$
T=\frac{\left|E_{2}^{\prime}\right|^{2}}{\left|E_{1}\right|^{2}}=\sin ^{2}\left(\frac{\pi \Delta n L}{\lambda}\right)=\frac{\left|M_{C C W}-M_{C W}\right|^{2}}{4}
$$

In addition, the variation of transmission spectra at independent operating wavelength can also be obtained using the following equation [20]

$$
T=\frac{1}{16} \|\left.\left[\begin{array}{cc}
-K_{1} \cdot e^{i \Delta \varphi_{1}} & K_{2} e^{i \Delta \varphi_{2}}+K_{3} e^{i\left(\Delta \varphi_{1}+\Delta \varphi_{2}\right)}+K_{4} e^{i \Delta \varphi_{1}}+K_{5} \\
K_{6}+K_{7} e^{i \Delta \varphi_{1}}+K_{8} e^{i \Delta \varphi_{2}}+K_{9} e^{i\left(\Delta \varphi_{1}+\Delta \varphi_{2}\right)} & -K_{1} \cdot e^{i \Delta \varphi_{2}}
\end{array}\right]\right|^{2}
$$

Here, $K_{1}$ until $K_{9}$ are expressed as

$$
\begin{aligned}
& K_{1}=2 \cdot i \cdot\left[\left(\sin \left(2 \theta_{12}\right)+\sin \left(2 \theta_{12}-2 \theta_{13}-2 \theta_{11}\right)\right) \cdot\left(\sin \left(2 \theta_{22}-2 \theta_{23}\right)-\sin \left(2 \theta_{22}-2 \theta_{21}\right)\right)\right. \\
& \left.+\left(\sin \left(2 \theta_{22}\right)+\sin \left(2 \theta_{22}-2 \theta_{23}-2 \theta_{21}\right)\right) \cdot\left(\sin \left(2 \theta_{12}-2 \theta_{11}\right)-\sin \left(2 \theta_{12}-2 \theta_{13}\right)\right)\right] \\
& K_{2}=\left[\cos \left(2 \theta_{12}\right)-\cos \left(2 \theta_{12}-2 \theta_{13}-2 \theta_{11}\right)+i \cdot\left(\cos \left(2 \theta_{12}-2 \theta_{13}\right)+\cos \left(2 \theta_{12}-2 \theta_{11}\right)\right)\right] \\
& \cdot\left[\sin \left(2 \theta_{22}\right)+\sin \left(2 \theta_{22}-2 \theta_{23}-2 \theta_{21}\right)+i \cdot\left(\sin \left(2 \theta_{22}-2 \theta_{21}\right)-\sin \left(2 \theta_{22}-2 \theta_{23}\right)\right)\right] \\
& K_{3}=\left[\sin \left(2 \theta_{12}\right)+\sin \left(2 \theta_{12}-2 \theta_{13}-2 \theta_{11}\right)+i \cdot\left(\sin \left(2 \theta_{12}-2 \theta_{11}\right)-\sin \left(2 \theta_{12}-2 \theta_{13}\right)\right)\right] \\
& \cdot\left[\cos \left(2 \theta_{22}-2 \theta_{23}-2 \theta_{21}\right)-\cos \left(2 \theta_{12}\right)+i \cdot\left(\cos \left(2 \theta_{12}-2 \theta_{11}\right)+\cos \left(2 \theta_{22}-2 \theta_{23}\right)\right)\right] \\
& K_{4}=\left[\sin \left(2 \theta_{23}+2 \theta_{21}-2 \theta_{22}\right)-\sin \left(2 \theta_{22}\right)+i \cdot\left(\sin \left(2 \theta_{22}-2 \theta_{23}\right)-\sin \left(2 \theta_{22}-2 \theta_{21}\right)\right)\right] \\
& \cdot\left[\cos \left(2 \theta_{12}-2 \theta_{13}-2 \theta_{11}\right)-\cos \left(2 \theta_{12}\right)+i \cdot\left(\cos \left(2 \theta_{12}-2 \theta_{11}\right)+\cos \left(2 \theta_{12}-2 \theta_{13}\right)\right)\right] \\
& K_{5}=\left[\sin \left(2 \theta_{13}+2 \theta_{11}-2 \theta_{12}\right)-\sin \left(2 \theta_{12}\right)+i \cdot\left(\sin \left(2 \theta_{12}-2 \theta_{13}\right)-\sin \left(2 \theta_{12}-2 \theta_{11}\right)\right)\right] \\
& \cdot\left[\cos \left(2 \theta_{22}\right)-\cos \left(2 \theta_{22}-2 \theta_{23}-2 \theta_{21}\right)+i \cdot\left(\cos \left(2 \theta_{22}-2 \theta_{23}\right)+\cos \left(2 \theta_{22}-2 \theta_{21}\right)\right)\right] \\
& K_{6}=\left[\sin \left(2 \theta_{12}\right)+\sin \left(2 \theta_{12}-2 \theta_{13}-2 \theta_{11}\right)+i \cdot\left(\sin \left(2 \theta_{12}-2 \theta_{13}\right)-\sin \left(2 \theta_{12}-2 \theta_{11}\right)\right)\right] \\
& \cdot\left[\cos \left(2 \theta_{22}\right)-\cos \left(2 \theta_{22}-2 \theta_{23}-2 \theta_{21}\right)+i \cdot\left(\cos \left(2 \theta_{22}-2 \theta_{23}\right)+\cos \left(2 \theta_{22}-2 \theta_{21}\right)\right)\right] \\
& K_{7}=\left[\cos \left(2 \theta_{12}-2 \theta_{13}-2 \theta_{11}\right)-\cos \left(2 \theta_{12}\right)+i \cdot\left(\cos \left(2 \theta_{12}-2 \theta_{13}\right)+\cos \left(2 \theta_{12}-2 \theta_{11}\right)\right)\right] \\
& \cdot\left[\sin \left(2 \theta_{22}\right)+\sin \left(2 \theta_{22}-2 \theta_{23}-2 \theta_{21}\right)+i \cdot\left(\sin \left(2 \theta_{22}-2 \theta_{23}\right)-\sin \left(2 \theta_{22}-2 \theta_{21}\right)\right)\right] \\
& K_{8}=\left[\cos \left(2 \theta_{12}-2 \theta_{13}-2 \theta_{11}\right)-\cos \left(2 \theta_{12}\right)-i \cdot\left(\cos \left(2 \theta_{12}-2 \theta_{13}\right)+\cos \left(2 \theta_{12}-2 \theta_{11}\right)\right)\right] \\
& \cdot\left[\sin \left(2 \theta_{22}\right)+\sin \left(2 \theta_{22}-2 \theta_{23}-2 \theta_{21}\right)+i \cdot\left(\sin \left(2 \theta_{22}-2 \theta_{23}\right)-\sin \left(2 \theta_{22}-2 \theta_{21}\right)\right)\right] \\
& K_{9}=\left[\sin \left(2 \theta_{13}+2 \theta_{11}-2 \theta_{12}\right)-\sin \left(2 \theta_{12}\right)+i \cdot\left(\sin \left(2 \theta_{12}-2 \theta_{11}\right)-\sin \left(2 \theta_{12}-2 \theta_{13}\right)\right)\right] \\
& \cdot\left[\cos \left(2 \theta_{22}-2 \theta_{23}-2 \theta_{21}\right)-\cos \left(2 \theta_{22}\right)+i \cdot\left(\cos \left(2 \theta_{22}-2 \theta_{23}\right)+\cos \left(2 \theta_{22}-2 \theta_{21}\right)\right)\right]
\end{aligned}
$$


From (11) and (12), the transmission $T$ consist of many simple combinatorial parameters, which are the products of the phase modulation $\left(e^{i \Delta \varphi 1}, e^{i \Delta \varphi 2}\right.$ or $\left.e^{i(\Delta \varphi 1+\Delta \varphi 2)}\right)$ and the amplitude modulations $\left(K_{1}\right.$ to $\left.K_{9}\right)$.

\section{RESULTS AND DISCUSSION}

In order to characterize the two-stage Sagnac loop filter (SLF), the transmission spectrum of the structure shown in Figure 1 is simulated by adjusting the PMF parameters $(L$ and $\Delta n)$ and PC parameters $\left(\theta_{11}\right.$, $\theta_{12}, \theta_{13}, \theta_{21}, \theta_{22}$, and $\theta_{23}$ ). When the PMF with the following parameters: $L_{1}=10 \mathrm{~m}, \Delta n_{1}=1 \times 10^{-4}, L_{2}=5 \mathrm{~m}$, and $\Delta n_{2}=4 \times 10^{-4}$ are applied, the changes of PC parameters will cause the transmission-amplitude remodulation.

In Figure 2, several transmission spectra from $1544 \mathrm{~nm}$ to $1556 \mathrm{~nm}$ are plotted with its PCs states changed for every plate. Each figure contains six different values of polarization angle based on plates of PC1 and PC2. Figure 2(a) shows the three wavelength peaks when the PC1 and PC2 states at $\theta_{11}=0.415 \pi$, $\theta_{12}=0.02 \pi, \theta_{13}=0.36 \pi, \theta_{21}=0.64 \pi, \theta_{22}=0.05 \pi$, and $\theta_{23}=0.654 \pi$. If continuously adjusting the PCs, as shown in Figure $2(\mathrm{~b})\left(\theta_{11}=0.44 \pi, \theta_{12}=0.0001 \pi, \theta_{13}=0.0001 \pi, \theta_{21}=0.635 \pi, \theta_{22}=0.035 \pi\right.$, and $\left.\theta_{23}=0.76 \pi\right)$ and Figure 2 (c) $\left(\theta_{11}=0.47 \pi, \theta_{12}=0.0001 \pi, \theta_{13}=0.01 \pi, \theta_{21}=0.555 \pi, \theta_{22}=0.035 \pi\right.$, and $\left.\theta_{23}=0.61 \pi\right)$, the left wavelength peaks of the multiple dual wavelength filtering channel declines gradually and is becoming multiple single wavelength channels condition.

From Figure 2(d), when the polarization angle of $\theta_{11}, \theta_{12}, \theta_{13}, \theta_{21}, \theta_{22}$, and $\theta_{23}$, is set to $0.82 \pi, 0.01 \pi$, $0.7 \pi, 0.15 \pi, 0.05 \pi$, and $0.6 \pi$, a flat multiwavelength spectrum is obtained. Multiple single wavelength condition is observed in Figure 2(d) where each channel contains only single wavelength peak. From Figure 2(a) to Figure 2(d), the results show that by adjusting the states of the PCs, the transmission spectrum of the two-stage SLF can be switch from multiple triple-wavelength to multiple dual-wavelength or even to multiple single-wavelength.
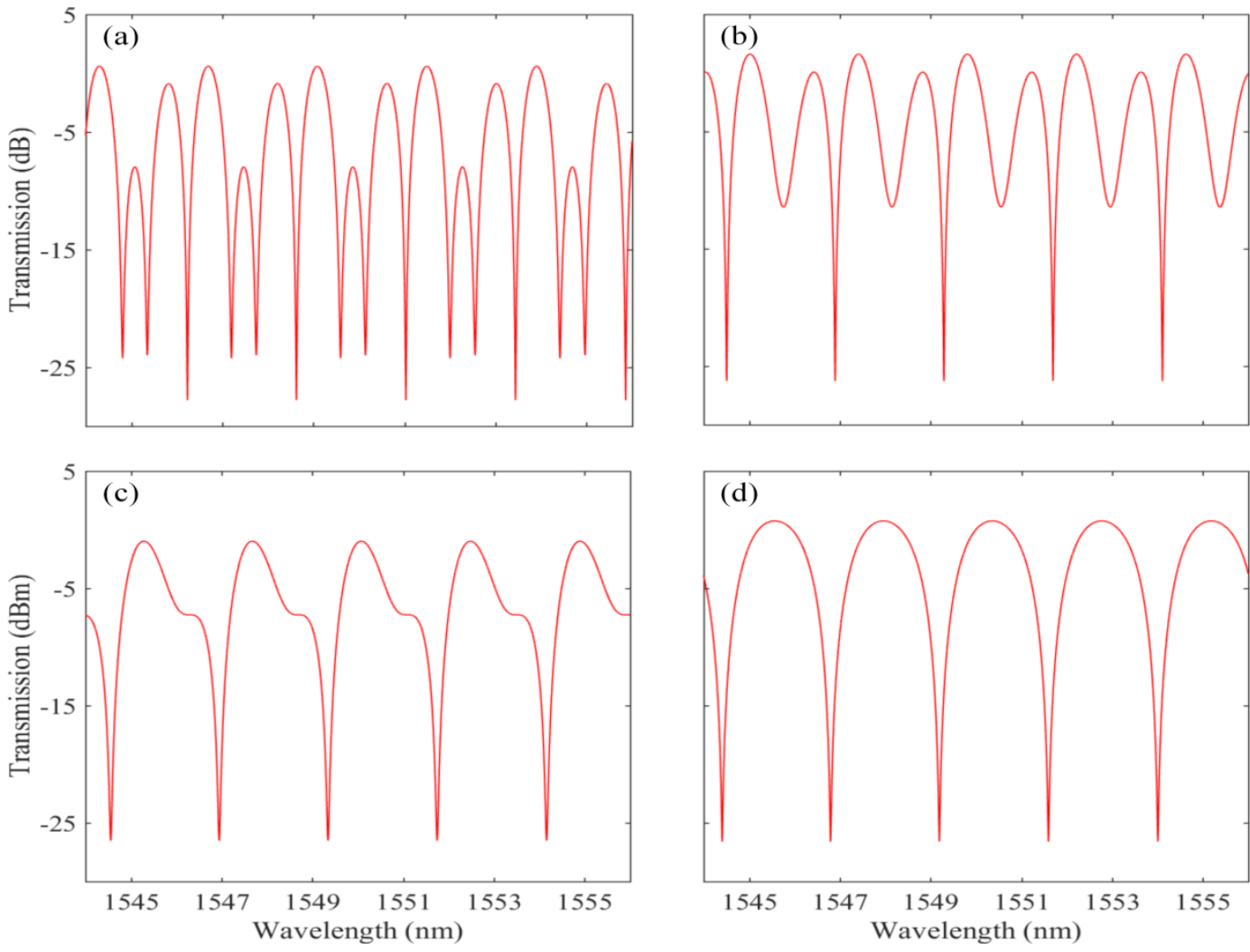

Figure 2. The variation of transmission spectra of the two-stage Sagnac loop filter (SLF) at different radian of PC1 and PC2 state; (a) $\theta_{11}=0.415 \pi, \theta_{12}=0.02 \pi, \theta_{13}=0.36 \pi, \theta_{21}=0.64 \pi, \theta_{22}=0.05 \pi$, and $\theta_{23}=0.654 \pi$, (b) $\theta_{11}=0.44 \pi, \theta_{12}=0.0001 \pi, \theta_{13}=0.0001 \pi, \theta_{21}=0.635 \pi, \theta_{22}=0.035 \pi$, and $\theta_{23}=0.76 \pi$,

(c) $\theta_{11}=0.47 \pi, \theta_{12}=0.0001 \pi, \theta_{13}=0.01 \pi, \theta_{21}=0.555 \pi, \theta_{22}=0.035 \pi$, and $\theta_{23}=0.61 \pi$ (d) $\theta_{11}=0.82 \pi$, $\theta_{12}=0.01 \pi, \theta_{13}=0.7 \pi, \theta_{21}=0.15 \pi, \theta_{22}=0.05 \pi$, and $\theta_{23}=0.6 \pi$ 
We then further investigating the two-stage Sagnac loop filter (SLF) characterizations by altering the PMFs length $\left(L_{1}\right.$ and $\left.L_{2}\right)$. The transmission spectrum as shown in Figure 3 are simulated with the birefringence of the PMFs and PCs fixed with the following parameters: $\Delta n_{1}=1 \times 10^{-4}, \Delta n_{2}=4 \times 10^{-4}$, $\theta_{11}=0.82 \pi, \theta_{12}=0.01 \pi, \theta_{13}=0.7 \pi, \theta_{21}=0.15 \pi, \theta_{22}=0.05 \pi$, and $\theta_{23}=0.6 \pi$.

In Figure 3, several transmission spectra from $1544 \mathrm{~nm}$ to $1556 \mathrm{~nm}$ are plotted with the condition of $L_{1}<L_{2}, L_{1}>L_{2}$ and $L_{1}=L_{2}$. Figure 3(a) and Figure 3(b) contains two different values of PMF1 and PMF2 length while Figure 3(c) and Figure 3(d) have same values of PMF1 and PMF2 length. Figure 3(a) gives the transmission spectrum for $L_{1}<L_{2}$, (in this case $L_{1}=5 \mathrm{~m}$ and $L_{2}=10 \mathrm{~m}$ ). Each channel consists of multiple wavelength peaks and minimum wavelength interval was observed. In Figure 3(b), a maximum wavelength interval can be obtained when the length of PMFs are $L_{1}=10 \mathrm{~m}$ and $L_{2}=5 \mathrm{~m}$. Based on this calculation, the best multiple single-wavelength spectrum can be achieved when the length of $L_{1}$ is longer than $L_{2}$.

Finally, in Figure 3(c) and Figure 3(d), identical length was set for the PMF1 and PMF2. The length of both PMFs in Figure 3(c) are $L_{1}=5 \mathrm{~m}$ and $L_{2}=5 \mathrm{~m}$, while in Figure 3(d) the length of both PMFs are increased to $L_{1}=10 \mathrm{~m}$ and $L_{2}=10 \mathrm{~m}$. It is observed that the bandwidth is becoming narrower as both of the PMFs length increases. From results represented in Figure 2 and Figure 3, it can be noted that the given PMFs and the PCs parameters gives significant influence to the wavelength interval of the SLF.
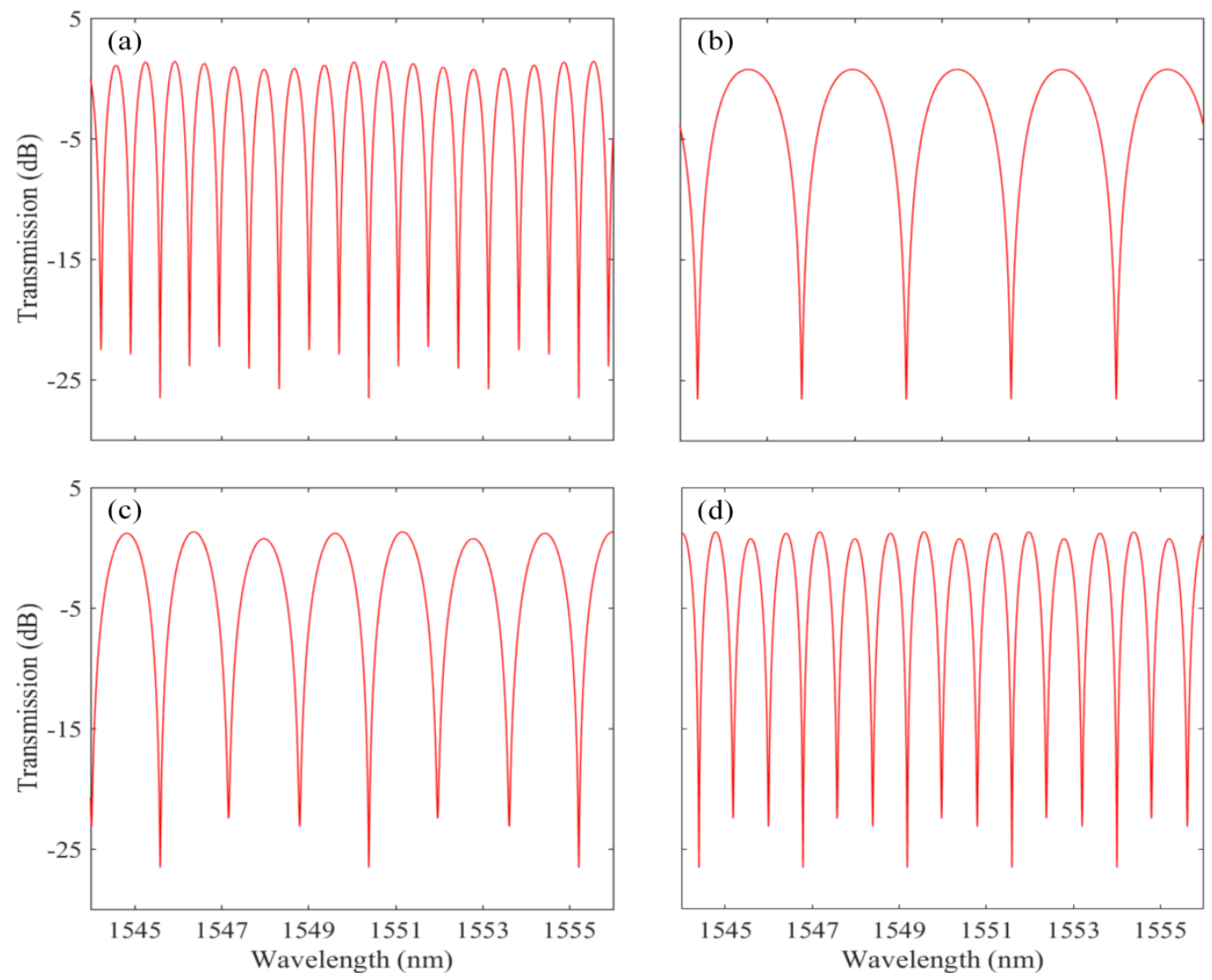

Figure 3. The variation of transmission spectra of the two-stage Sagnac loop filter (SLF) at different length of PMF1 and PMF2; (a) $L_{1}=5 \mathrm{~m}$ and $L_{2}=10 \mathrm{~m}$ (b) $L_{1}=10 \mathrm{~m}$ and $L_{2}=5 \mathrm{~m}$ (c) $L_{1}=5 \mathrm{~m}$ and $L_{2}=5 \mathrm{~m}$ (d) $L_{1}=10 \mathrm{~m}$ and $L_{2}=10 \mathrm{~m}$

\section{CONCLUSION}

This study discussed the theoretical analysis of the two-stage Sagnac loop filter (SLF) where the characteristic was analyzed using the Jones matrix. The two-stage SLF consist of two polarization maintaining fibers (PMFs) and two polarization controllers (PCs). Multiple single, dual and triple wavelengths were observed by adjusting the polarization angle of the PCs. The wavelength interval is depending on both of the PMFs and the PCs parameters. Maximum wavelength interval was obtained when the length of PMF1 is made longer than the length of PMF2. In addition, the two-stage SLF achieved its flat 
multi-wavelength when the PMFs $\left(L_{1}=10 \mathrm{~m}\right.$ and $\left.L_{2}=5 \mathrm{~m}\right)$ and PCs $\left(\theta_{11}=0.82 \pi, \theta_{12}=0.01 \pi, \theta_{13}=0.7 \pi\right.$, $\theta_{21}=0.15 \pi, \theta_{22}=0.05 \pi$, and $\left.\theta_{23}=0.6 \pi\right)$ are applied.

\section{ACKNOWLEDGEMENTS}

This work was supported in part by the Ministry of Higher Education Malaysia (MOHE) under Fundamental Research Grant Scheme (FRGS) Vot 1622 and Research Acculturation Collaborative Effort (RACE) Vot 1509.

\section{REFERENCES}

[1] W. He, L. Zhu, M. Dong, and F. Luo, "Tuneable and stable multi-wavelength thulium-doped ring-cavity fibre laser based on Sagnac loop and Mach-Zehnder filter utilizing thin-core fibre,” Laser Physics, vol. 26, p. 125102, 2016.

[2] B. Wu, M. Wang, Y. Tang, J. Sun, J. Zhang, F. Yan, and S. Jian, "Optical single sideband modulation with tunable optical carrier-to-sideband ratio using a modulator in a Sagnac loop," Optics and Laser Technology, vol. 91, pp. 98-102, 2017.

[3] X. Wang, Y. Zhu, P. Zhou, X. Wang, H. Xiao, and L. Si, "Tunable, multiwavelength Tm-doped fiber laser based on polarization rotation and four-wave mixing effect," Optics Express, vol. 21, pp. 25977-25984, 2013.

[4] H. Lin, Y. F. Huang, and Y. S. Huang, "Full L-band coverage of multiwavelength erbium-doped fiber laser," Optics Communications, vol. 284, pp. 5357-5360, 2011.

[5] Y. Li, J. Tian, M. Quan, and Y. Yao, "Tunable multiwavelength Er-doped fiber laser with a two-stage Lyot filter," IEEE Photonics Technology Letter, vol. 29, pp. 287-290, 2017.

[6] H. Xie, J. Sun, D. Feng, and L. Qian, "Compact Multiwavelength Brillouin Fiber Laser by Utilizing EDF as Hybrid Gain Media," IEEE Photonics Journal, vol. 7, p. 1504110, 2015.

[7] X. Liu, L. Zhan, S. Luo, Z. Gu, J. Liu, Y. Wang, and Q. Shen, "Multiwavelength erbium-doped fiber laser based on a nonlinear amplifying loop mirror assisted by un-pumped EDF," Optics Express, vol. 20, pp. 7088-7094, 2012.

[8] H. Ahmad, M. Z. Zulkifli, M. H. Jemangin, and S. W. Harun, "Distributed feedback multimode Brillouin-Raman random fiber laser in the S-band," Laser Physics Letters, vol. 10, p. 055102, 2013.

[9] G. Sun, Y. Zhou, L. Cui, and Y. Chung, "Tunable multiwavelength SOA-fiber ring laser based on sagnac loop mirror incorporating few-mode high birefringence fiber", Laser Physics, vol. 21, pp. 1899-1902, 2011.

[10] N. A. B. Ahmad, S. H. Dahlan, and N. A. Cholan, "Experimental investigation of the residual waves in a multiwavelength Brillouin-erbium fiber laser, " IEEE $6^{\text {th }}$ International Conference on Photonics, ICP 2016, p. $7510021,2016$.

[11] Z. X. Zhang, Z. Q. Ye, M. H. Sang, and Y. Y. Nie, "Nonlinear-polarization-rotation based multiwavelength erbium-doped fiber lasers with highly nonlinear fiber," Laser Physics, vol. 21, pp. 1820-1824, 2011.

[12] Y. Wang, L. Pei, J. Li, and Y. Li, "All optical multi-wavelength single-sideband modulated WDM radio-over-fiber systems by introducing a Sagnac loop filter," Optical Fiber Technology, vol. 32, pp. 36-42, 2016.

[13] L. Hou, M. Li, X. He, Q. Lin, H. Guo, B. Lu, X. Qi, H. Chen, and J. Bai, "Wavelength-tunable dissipative pulses from Yb-doped fiber laser with Sagnac filter," Laser Physics Letter, vol. 13, p. 125302, 2016.

[14] T. Wang, X. Miao, X. Zhou, and S. Qian, "Tunable multiwavelength fiber laser based on a double Sagnac HiBi fiber loop," Applied Optics, vol. 51, pp. C111-C116, 2012.

[15] J. Jung, and Y. W. Lee, "Tunable fiber comb filter based on simple waveplate combination and polarizationdiversified loop," Optics and Laser Technology, vol. 91, pp. 63-70, 2017.

[16] L. K. Lee, M. P. Fok, S. M. Wan, and C. Shu, "Optically controlled Sagnac loop comb filter," Optics Express, vol. 12, pp. 6335-6340, 2004.

[17] J. Wang, K. Zheng, J. Peng, L. Liu, J. Li, and S. Jian, "Theory and experiment of a fiber loop mirror filter of twostage polarization-maintaining fibers and polarization controllers for multiwavelength fiber ring laser," Optics Express, vol. 17, pp. 10573-10583, nm2009.

[18] S. Feng, Q. Mao, L. Shang, and J. W. Y. Lit, "Reflectivity characteristics of the fiber loop mirror with a polarization controller," Optics Communications, vol. 277, pp. 322-328, 2007.

[19] F. Heismann, "Analysis of a Reset-Free Polarization Controller for Fast Automatic Polarization Stabilization in Fiber-optic Transmission Systems," Journal of Lightwave Technology, vol. 12, pp. 690-699, 1994.

[20] M. Zhou, Z. Luo, Z. Cai, C. Ye, H. Xu, and J. Wang, "Switchable and tunable multiple-channel erbium doped fiber laser using graphene-polymer nanocomposite and asymmetric two stage fiber Sagnac loop filter," Applied Optics, vol. 50, pp. 2940-2948, 2011. 


\section{BIOGRAPHIES OF AUTHORS}
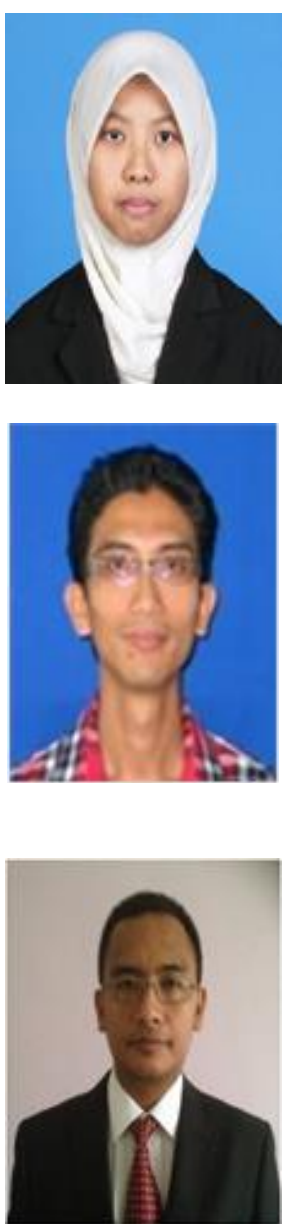

Nurul Atiqah Bt Ahmad received the Bachelor and Master degree in Electrical and Electronic Engineering from the University Tun Hussein Onn Malaysia (UTHM), in 2013 and 2015. She is a Graduate Resesarch Assistant. Currently she is pursuing the PhD degree with the Research Center for Applied Electromagnetics (EMCenter, UTHM). Her research interests include multiwavelength fiber laser and microwave photonics.

Samsul Haimi Dahlan received his $\mathrm{PhD}$ degree in Signal Processing and Telecommunications from the Universite de Rennes 1, France, in 2012. He is a Senior Lecturer with the Faculty of Electric and Electronic Engineering, Universiti Tun Hussein Onn Malaysia (UTHM) since March 2012. Currently he is with the Research Center for Applied Electromagnetics (EMCenter, UTHM) as the principal researcher and appointed as the Head of the center since April 2015. He has authored and co-authored numbers of journals including the IEEE TRANSACTION on ELECTROMAGNETIC COMPATIBILITY and IEEE AWPL. His research interest includes Optical-Microwave generator, focusing system (dielectric lens and transmitarray's synthesis), computational electromagnetic technique namely the BOR-FDTD and material characterizations. He is supervising a numbers of PhD's, master's, and bachelor's students and involved in several research projects sponsored by the industry and government agencies.

Noran Azizan Cholan was born on 31st August 1979 in Segamat, Johor, Malaysia. He received his bachelor degree in Electronics Engineering from Universiti Tenaga Nasional (UNITEN), Malaysia in 2002. Afterwards in 2004, he obtained his master degree in ElectronicsTelecommunications Engineering form Universiti Teknologi Malaysia (UTM), Malaysia. In 2010, he enrolled as a PhD student in Universiti Putra Malaysia under the supervision of Prof. Dr. Mohd Adzir Mahdi. During his PhD study in 2012, he went to Swansea University, UK for a 3 months attachment under the guidance of Prof. Emiritus Michel E. Marhic for a collaborated project of Brillouin amplifiers. Later in the same year, he went to The Hong Kong Polytechnic University, Hong Kong for a month to collaborate with Prof Lu Chao for a project of optical communication systems. Since then, he has served as a senior lecturer in Universiti Tun Hussein Onn Malaysia (UTHM). As of now, he has been authors/co-authors for 8 journals and 14 conference proceeding papers. 